\title{
Anastomotic leakage rate following modified double staple technique of anterior resection with vertical division of the rectum for rectal cancer surgery
}

Masahiro Tsubaki*, Yasuko Suzuki, Mio Adachi, Takaaki Watanabe, Ko Kurimori, Naoya Ikeda, Kenichiro Yoshitake, Yawara Omd Hiroshi Nakamura, Yuji Kumasiro, Masaaki Kanenobu and Syoichi Kato

Department of Surgery, Yuai Memorial Hospital, 770, Higashi-ushigaya, Koga, Ibaraki, Japan

\begin{abstract}
Introduction: Anastomotic leakage (AL) after double staple technique (DST) for rectal cancer surgery is a major complication. The aim of this study is to assess the usefulness of the modified double-stapling technique with vertical division of the rectum (IO-DST).

Patients and methods: Forty-three consecutive cases performed by IO-DST technique from July 2011 to August 2015 were reviewed retrospectively. All cases were performed by IO-DST technique for the anastomosis controlled by one skillful colorectal surgeon who has worked for over 30 years as a surgeon and over 20 years as a colorectal surgeon, experienced in over 1500 cases of colorectal cancer as operator or an assistant. AL was clinically evaluated.

Results: 41 cases (95.3\%) were performed in open surgery, left colic artery (LCA) was preserved in 35 cases (81.4\%), diverting ileostomy was performed in 6 cases (14\%) and pelvic lymphadenectomy was performed in 7 cases (16.3\%). IO-DST anastomosis was performed in 30 cases (69.8\%) with PCEEA 31mm. Leakage was found in one case $(2.3 \%)$, it was not found for the cases of anterior resection or the cases with preservation of LCA.
\end{abstract}

Conclusion: We conclude IO-DST anastomosis for rectal cancer was safe procedure with low AL rate.

\section{Introduction}

Anastomotic leakage (AL) after double staple technique (DST) for rectal cancer surgery is a major complication. The modified doublestapling technique with vertical division of the rectum (IO-DST) has

been reported as a feasible and safe procedure during the rectal cancer surgery by Maeda et.al., [1]. According to that report, 80 consecutive cases reconstructed by IO-DST were reviewed in my previous study [2]. Leakage was found in 7 cases, it was $7.5 \%$. The aim of this study is to assess the usefulness of this technique.

\section{Patients and methods}

Forty-three consecutive cases performed by IO-DST technique from July 2011 to August 2015 were reviewed retrospectively. The characteristics of patients were shown in Table 1. Location of the cancers were described according to the Japanese Classification of colorectal carcinoma [3]. Preoperative chemotherapy or radiotherapy was not performed.

All cases were performed by IO-DST technique for the anastomosis controlled by one skillful colorectal surgeon who has worked for over 30 years as a surgeon and over 20 years as a colorectal surgeon, experienced in over 1500 cases of colorectal cancer as operator or an assistant.

\section{Surgical procedure}

Patients took lithotomy position with heads slightly down. After detection at the root of the IMA, we preserved LCA as much as possible with dissection of the lymph-nodes at the root of the IMA. When we identified the positive lymph-nodes surrounding IMA, it was ligated completely at its root. After mobilized sigmoid colon and rectum with complete preserving superior hypo-gastric plexus, hypo-gastric plexus and pelvic plexus, sigmoid colon was divided by Endo GIA ${ }^{\mathrm{sx}} 60 \mathrm{~mm}$ Articulating Medium/Thick Reload with Tri-Staple ${ }^{\mathrm{Tm}}$ Technology (Medtronic, Minneapolis, MN, USA) or ECHELON FLEX ENDOPATH $^{\star} 60 \mathrm{~mm}$ Stapler (ETHICON ENDO-SURGERY, LLC.,

Table 1. Characteristics of patients.

\begin{tabular}{|c|c|}
\hline \multicolumn{2}{|c|}{ Gender } \\
\hline Men & 28 patients \\
\hline Women & 15 patients \\
\hline \multicolumn{2}{|c|}{ Location of the carcinoma $^{1}$} \\
\hline $\mathrm{Rs} \mathrm{:}$ & 18 cases \\
\hline $\mathrm{Rb}:$ & 11 cases \\
\hline Average age and range : & 14 cases \\
\hline
\end{tabular}

${ }^{1}$ According to the Japanese Classification of Colorectal Carcinoma, location of tumor was defined. Rectosigmoid cancer was defined as the bowel at the level of between the promontrium and the lower margin of the second sacral vertebra. Middle rectal cancer was defined as the bowel at the level of between the lower margin of the second sacral vertebra and the peritoneal reflection. Lower rectal cancer was defined as the bowel at the level of below the peritoneal reflection.

Correspondence to: Dr. Masahiro Tsubaki, Department of Surgery, Yuai Memorial Hospital, 770, Higashi-ushigaya, Koga, Ibaraki, Japan, E-mail: tsubamasa1987@gmail.com

Key words: rectal cancer, double-stapling technique, anastomotic leakage

Received: May 26, 2017; Accepted: June 12, 2017; Published: June 18, 2017 
Tsubaki M (2017) Anastomotic leakage rate following modified double staple technique of anterior resection with vertical division of the rectum for rectal cancer surgery

Cincinnati, OH, USA) and appropriately sized circular stapler was fitted in the proximal bowel. An occlusion clamp was placed vertically across the rectum distal to the tumor following washout of the remnant rectum using $2000 \mathrm{ml}$ warmed saline. Endo $\mathrm{GIA}^{\mathrm{max}} 60 \mathrm{~mm}$ Articulating Medium/Thick Reload with Tri-Staple ${ }^{\mathrm{Tu}}$ Technology or ECHELON 60 $\mathrm{mm}$ Gold Reload was applied vertically across the rectum distal to the bowel clamp and fired at an appropriate surgical margin one or two times in order to perform the IO-DST anastomosis.

According to the Japanese Society for Cancer of the Colon and Rectum (JSCCR) guidelines for the Treatment of Colorectal Cancer [4], pelvic lymphadenectomy (PL) was performed for the cancer which lowest margin was below the perinetoneal reflection or lower rectal cancer with over T3 and or lymph node metastasis that was preoperatively or intraoperatively diagnosed. PL was performed after resection of the cancer.

Finally Premium Plus CEEA ${ }^{\mathrm{m}}$ Staplers (PCEEA) (Medtronic, Minneapolis, MN, USA), usually $31 \mathrm{~mm}$ or Proximate ${ }^{\circledR}$ Intraluminal Stapler $(\mathrm{CDH}) 29 \mathrm{~mm}$ was perianally introduced and double-stapling anastomosis was performed. Diverting stoma was created when the anastomotic site was located within $5 \mathrm{~cm}$ from the anal verge. All cases were performed with an operator or first assistant, who had over 20 years' experience as a colorectal surgeon. AL was clinically evaluated.

\section{Results}

Operative methods were shown in Table 2. 41 cases (95.3\%) were performed in open surgery, LCA was preserved in 35 cases $(81.4 \%)$, diverting ileostomy was performed in 6 cases (14\%) and PL was performed in 7 cases (16.3\%). IO-DST anastomosis was performed in 30 cases (69.8\%) with PCEEA $31 \mathrm{~mm}$ (Table 3). The Duke's classification in each case is shown in Table 4. Leakage was found in one case $(2.3 \%)$, it was not found for the cases of AR and the cases with preservation of LCA (Table 5).

\section{Discussion}

$\mathrm{AL}$ was a major complication after AR or LAR for rectal cancer. Recently, after Laparoscopic Low Anterior Resection (LLAR) AL occurred $12.3 \%$ among 154 patients [5]. To prevent AL after LLAR, Tetsuo Ito reported the usefulness of transanal tube [6]. They reported that $\mathrm{AL}$ rate was $3.6 \%$ in 28 patients with tube in comparison with $24.4 \%$ without tube replacement. The other two studies reported that procedure was effective and safe to prevent $\mathrm{AL}$ after colorectal cancer surgery with DST reconstruction $[7,8]$. Generally, to perform DST technique, the rectum was divided horizontally. The rectum was

Table 2. Operative methods.

\begin{tabular}{|c|c|}
\hline Anterior resection (AR) & 11 cases \\
\hline Laparoscopic AR & 2 cases \\
\hline Low anterior resection (LAR) & 30 cases \\
\hline Preservation of left colic artery(LCA) & 35 cases \\
\hline Ligation of the inferior mesenteric artery (IMA) & 8 cases \\
\hline With covering ileostomy & 6 cases \\
\hline Without covering ileostomy & 37 cases \\
\hline With pelvic lymphadenectomy (PL) & 7 cases \\
\hline Without PL & 36 cases \\
\hline
\end{tabular}

Table 3. Types of stapler.

\begin{tabular}{|c|c|}
\hline PCEEA $28 \mathrm{~mm}$ & 6 cases \\
\hline PCEEA $31 \mathrm{~mm}$ & 30 cases \\
\hline CDH $29 \mathrm{~mm}$ & 7 cases \\
\hline
\end{tabular}

Table 4. Duke's classification of cancers

\begin{tabular}{|c|c|}
\hline Duke's A & 17 cases \\
\hline Duke's B & 13 cases \\
\hline Duke's C & 11 cases \\
\hline Duke's D & 2 cases \\
\hline
\end{tabular}

Table 5. The number of the patients who has been found leakage.

\begin{tabular}{|c|c|}
\hline Total number of the patients & $2.3 \%(1 / 43)$ \\
\hline AR & $0 \%$ \\
\hline LAR & $3.3 \%(1 / 30)$ \\
\hline Preservation of LCA & $0 \%$ \\
\hline Ligation of IMA & $12.5 \%(1 / 8)$ \\
\hline
\end{tabular}

divided vertically when IO-DST was performed. AL rate following this technique was reported to occur $2.9 \%$ of patients by Sato [9]. According to this report, $\mathrm{AL}$ rate of consecutive eighty cases of rectal tumor performed by IO-DST was $7.5 \%$ in my previous study [2]. AL was found 6 cases of 54 LAR cases (11.1\%). However, 21 cases of LAR with preservation of LCA were not found to occur AL. Therefore, we preserved LCA when we performed AR or LAR with IO-DST in this current study of consecutive 43 cases of rectal cancer. Only one case (2.3\%) was found to occur AL in this study. It was almost the same rate of the previous report of Dr. Sato [9] and was better than the report of Dr. Ito [6].

Ligation at the root of IMA was performed for the cases of $\mathrm{AL}$ in our previous study and this study. We considered that procedure would be one risk factor of AL. The preservation of the LCA in LLAR was reported to be associated with lower risk of AL [10]. It will be able to keep the blood supply to the proximal side of anastomosis and to have an enough length of the colon for the anastomosis for reducing anastomotic tension.

Kenji Kawada found AL 19 cases of 154 rectal cancer patients (12.3\%) [5]. They analyzed the tumor size and the recompression before stapler firing was risk factors for $\mathrm{AL}$ after laparoscopic LAR with DST anastomosis. He also reviewed preoperative, intraoperative and postoperative risk factors for anastomotic leakage after laparoscopic low anterior resection with DST anastomosis [11]. Multiple staple firing and diameter of circular stapler are intraoperative risk factors. Our one case occurring AL was 49 years old woman with high ligation of IMA and using PCEEA $31 \mathrm{~mm}$ for anastomosis. Before anastomosis, two times firing stapler was done to divide the rectum. Surgical specimen showed the tumor size was $6.5 \mathrm{~cm}$ and we could not pass the tumor by preoperative colonoscopy. Mechanical preparation was not enough for this case. She had several risk factors for AL.

\section{Conclusion}

We conclude AL rate following after IO-DST anastomosis was low. IO-DST anastomosis could be a safe procedure for reconstruction of anterior resection for rectal cancer.

\section{References}

1. Maeda K, Maruta M, Utsumi T, Okamura Y(1999) Vertical division of the rectum by endostapler in very low colorectal anastomosis with a double-stapling technique. Min Invas Ther \& Allied Technol.8: 3-4.

2. Tsubaki M, Ito Y, Fujita M, Kato H (2012) Use of the modified double-stapling technique with vertical division of the rectum during a sphincter-preserving operation for the treatment of a rectal tumor. Asian Journal of Surgery, 35:110-112. [Crossref]

3. Japanese Society for Cancer of the Colon and Rectum (2009) Japanese Classification of Colorectal Carcinoma. 1st English ed. Tokyo: Kanehara \& Co., Ltd. 
4. Watanabe T, Muro K, Ajioka Y, Hashiguchi Y, Ito Y, et al. (2017) Japanese Society for Cancer of the Colon and Rectum. Japanese Society for Cancer of the Colon and Rectum Guidelines 2016 for the Treatment of Colorectal Cancer. Int J Clin Oncol. [Crossref]

5. Kawada K, Hasegawa S, Hida K, Hirai K, Okoshi K, et al. (2014) Risk factors for anastomotic leakage after laparoscopic low anterior resection with DST anastomosis. Surg Endosc28: 2988-2995.[Crossref]

6. Ito T, Obama K, Sato T, Matsuo K,Inoue H, et.al. (2017) Usefulness of transanal tube placement for prevention of anastomotic leakage following laparoscopic low anterior resection.Asian J Endosc Surg. 10:17-22.

7. Matsuda M, Tsuruta M, Hasegawa H, Okabayashi K, Kondo T, et.al. (2016) Transanal drainage tube placement to prevent anastomotic leakage following colorectal cancer surgery with double stapling reconstruction. Surg Today. 46:613-620. [Crossref]
8. Shigeta K, Okabayashi K, Baba H, Hasegawa H, Tsuruta M, et.al. (2016) A metaanalysis of the use of a transanal drainage tube to prevent anastomotic leakage after anterior resection by double-stapling technique for rectal cancer. Surg Endosc. 30:543550. [Crossref]

9. Sato H, Maeda K, Hanai T, Matsumoto M, Aoyama H, Matsuoka H, et al. (2006) Modified double-stapling technique in low anterior resection for lower rectal carcinoma. Surg Today36:30-36. [Crossref]

10. Hino T, Okajima M, Shinomura M, Egi H, Ohdan H et.al. (2013) Effect of left colonic artery preservation on anastomotic leakage in laparoscopic anterior resection for middle and low rectal cancer. World J Surg37: 2935-2943. [Crossref]

11. Kawada K, Sakai Y (2016) Preoperative, intraoperative and postoperative risk factors for anastomotic leakage after laparoscopic low anterior resection with double stapling technique anastomosis. World J Gastroenterol. 22:5718-5727. [Crossref]

Copyright: $\odot 2017$ Tsubaki M. This is an open-access article distributed under the terms of the Creative Commons Attribution License, which permits unrestricted use, distribution, and reproduction in any medium, provided the original author and source are credited. 